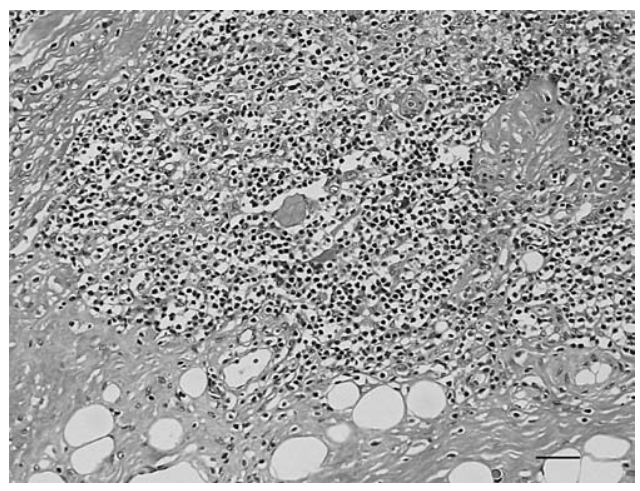

Figure 2. Pathologic findings of the resected thymoma. (Original magnification $200 \times$.) Intracapsular invasion and both small lymphocytic and epithelial components were observed. The patient was given a diagnosis of an invasive thymoma of mixed type.

\section{Discussion}

In patients with paraneoplastic SPS, surgical removal of the associating neoplasm is thought to be important in reducing neurologic symptoms. Sommer and colleagues ${ }^{5}$ previously reported that rats experienced spastic stiffness like human SPS with injection of purified IgG taken from a patient with paraneoplastic SPS associated with breast cancer. This suggests that paraneoplastic production of autoantibodies could be associated with paraneoplastic SPS.

Clinically, Hagiwara and associates ${ }^{4}$ and Tanaka and cowork$\mathrm{ers}^{3}$ reported SPS cases in which the painful stiffness was reduced by surgical resection of a thymoma. After resection, serum autoantibody titers should be reduced in such cases; however, in our patient the GAD antibody titer was once again increased 2 weeks after resection, despite a lack of symptoms. Moreover, as a result of traumatic stimulation, SPS suddenly relapsed in the clinically asymptomatic period after surgical intervention. This clinical course suggests that surgical removal of the tumor does not always mean the prevention of autoantibody productivity and is not always a curative treatment for paraneoplastic SPS, as with myasthenia gravis associated with thymoma.

\section{References}

1. Murinson BB. Stiff-person syndrome. Neurologist. 2004;10:131-7.

2. Nicholas AP, Chatterjee A, Arnold MM, Claussen GC, Zorn GL Jr, Oh SJ. Stiff-persons' syndrome associated with thymoma and subsequent myasthenia gravis. Muscle Nerve. 1997;20:493-8.

3. Tanaka $\mathrm{H}$, Matsumura A, Okumura $\mathrm{M}$, Kitaguchi M, Yamamoto S, Iuchi K. Stiff man syndrome with thymoma. Ann Thorac Surg. 2005;80:739-41.

4. Hagiwara H, Enomoto-Nakatani S, Sakai K, Ugawa Y, Kusunoki S, Kanazawa I. Stiff-person syndrome associated with invasive thymoma: a case report. J Neurol Sci. 2001;193:59-62.

5. Sommer C, Weishaupt A, Brinkhoff J, Biko L, Wessig C, Gold R, et al. Paraneoplastic stiff-person syndrome: passive transfer to rats by means of IgG antibodies to amphiphysin. Lancet. 2005;365:1406-11.

\title{
Postoperative pulmonary embolism detected with multislice computed tomography in lung surgery for cancer
}

\author{
Giuliano Daddi, MD, ${ }^{a}$ Giulia Milillo, MD, ${ }^{\mathrm{b}}$ Luciano Lupattelli, MD, ${ }^{\mathrm{c}}$ Mark Ragusa, MD, ${ }^{a}$ Angelo Lemmi, MD, \\ Francesco Puma, MD, ${ }^{d}$ and Giancarlo Agnelli, ${ }^{b}$ for the Pulmonary Embolism in Thoracic Surgery Study Group, Perugia, Italy
}

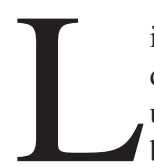
imited information is currently available on the incidence of venous thromboembolism (VTE) in patients undergoing lung surgery for cancer. ${ }^{1,2}$ Pulmonary embolism (PE) has been found to be a frequently fatal postoperative complication, as confirmed by autopsy in $15.2 \%$ of

From the Thoracic Surgery Unit, ${ }^{a}$ and Departments of Internal Medicine and Vascular Diseases ${ }^{\mathrm{b}}$ and Radiology, ${ }^{\mathrm{c}}$ Perugia University, Perugia, Italy; and Thoracic Surgery Unit, Perugia University at Terni, ${ }^{\mathrm{d}}$ Italy.

Presented as a poster at "Chest 2004," Annual Meeting of the American College of Chest Physicians, Seattle, Wash, October 23-28, 2004.

Address for reprints: Mark Ragusa, MD, S.C. Chirurgia Toracica, Università di Perugia, Ospedale Silvestrini, Via G. Dottori, 06156, Perugia, Italy (E-mail: drfastnet@bigfoot.com).

J Thorac Cardiovasc Surg 2006;132:197-8

$0022-5223 / \$ 32.00$

Copyright $\odot 2006$ by The American Association for Thoracic Surgery doi:10.1016/j.jtcvs.2006.03.036 postresectional deaths. ${ }^{3}$ The clinical burden of PE in surgical patients is underestimated, probably because the majority of VTE remains asymptomatic or associated with aspecific symptoms. Several factors increase the thromboembolic risk in patients undergoing lung cancer surgery: intrinsic procoagulant effect of cancer, extensive surgical intervention, dependent limb position in the operating room, and vessel injury consequent to the operation.

$\mathrm{PE}$ in patients undergoing thoracic surgery has some peculiarities. Indeed, in thoracic surgery, in addition to the established risk factors for VTE, local factors related to surgical technique, direct vascular injury, or both could play a remarkable role. ${ }^{4}$

The aim of this study was to assess the incidence of PE after lung surgery for cancer by using multislice computed tomographic (MSCT) scanning.

\section{Methods}

The Pulmonary Embolism in Thoracic Surgery Study was a prospective study performed to identify the incidence of PE by means of MSCT scanning after lung surgery for cancer. 
Table 1. Cancer histology

\begin{tabular}{lc}
\hline Histology & No. of patients \\
\hline Epidermoid carcinoma & $21(42 \%)$ \\
Adenocarcinoma & $14(28 \%)$ \\
Anaplastic & $4(8 \%)$ \\
Benign & $1(2 \%)$ \\
Metastatic & $2(4 \%)$ \\
Other types & $8(16 \%)$
\end{tabular}

MSCT scanning was performed with a 4-row computed tomogram (GE Light-Speed, $4 \times 1.25$ ); technical parameters were high speed, 2.5-mm collimation, $220 \mathrm{mAs}$, and $120 \mathrm{Kv}$. All examinations were separately reviewed by 2 different radiologists.

All patients underwent chest MSCT scanning within 7 to 15 days from the time of the operation. Diagnostic criteria for PE were as follows: (1) abrupt cutoff of vessels, often distally enlarged, in case of complete obstruction; (2) partial central vascular filling defect (known as "polo mint" or "tram lining" signs); and (3) partial eccentric vascular filling defect.

Patient data, including age, sex, history of VTE, previous major operation, chemotherapy, comorbidity, smoking habit, medications, type of lung resection, tumor size, histology, and stage, were considered potential risk factors for VTE.

Patients received pharmacologic prophylaxis for VTE with low-molecular-weight heparin, starting 12 to 24 hours after surgical intervention until discharge, at a dosage of 3000 to $4000 \mathrm{UI} / \mathrm{d}$.

The primary outcome of the study was PE incidence. The secondary aim was to analyze the correlation between surgical technique and the occurrence of thrombus in the pulmonary arterial stumps. The study protocol was approved by the regional institutional review board. All patients provided written informed consent.

\section{Results}

Fifty patients were included in this study. The average age was 66.5 years (range, 26-90 years). Twelve were women, and 38 were men. All but 2 patients received VTE prophylaxis.

Thirty-six patients underwent lobectomy, 12 underwent pneumonectomy, and 2 underwent wedge resection. The diagnosis of cancer was confirmed in all cases except one, a patient affected by tuberculosis.

Histology showed primary lung cancer in 47 patients. Details are summarized in Table 1.

Among the patients with primary lung carcinoma, 18 were in stage I, 14 were in stage II, and 15 were in stage III.

Seven (14\%) patients showed PE at MSCT scanning, 5 involving the central arteries (principal, lobar, and segmentary) and 2 involving the subsegmentary arteries. Among the 7 positive cases, 2 were in situ thrombosis of the residual vascular stump, one with direct extension to the contralateral pulmonary vasculature.

PE was bilateral in 2 patients. Two of the positive results at MSCT scanning were symptomatic, and only 1 had a clinically overt deep venous thrombosis confirmed by means of duplex
Table 2. Risk factors for pulmonary embolism after lung surgery

\begin{tabular}{lll}
\hline Risk factors & \multicolumn{1}{c}{ Incidence } \\
\hline Male vs female sex & $6 / 38$ & $(15.7 \%)$ vs $1 / 12(8.3 \%)$ \\
COPD vs no COPD & $4 / 14$ & $(28.6 \%)$ vs $3 / 36(8.3 \%)$ \\
Lobectomy vs pneumonectomy & $6 / 36$ & $(16.6 \%)$ vs $1 / 12(8.3 \%)$ \\
Lower lobectomy vs upper & $4 / 16$ & $(25.0 \%)$ vs $2 / 20(10.0 \%)$ \\
lobectomy & & \\
Right side vs left side & $3 / 20$ & $(15 \%)$ vs $4 / 30(13.3 \%)$ \\
Tumor $>3$ cm vs smaller tumors & $5 / 37$ & $(13.5 \%)$ vs $1 / 12(8.3 \%)$
\end{tabular}

$C O P D$, Chronic obstructive pulmonary disease.

scanning. Because of low diagnostic accuracy, duplex scanning was not performed in asymptomatic patients.

One patient died on postoperative day 10 of respiratory failure. The results of MSCT scanning were negative for PE. Risk factors for PE are summarized in Table 2.

\section{Discussion}

In this study the incidence of PE detected by means of MSCT scanning after lung surgery for cancer was $14 \%$, which is higher than the $5 \%$ previously reported on the basis of lung scanning. ${ }^{1}$

As in other series on postoperative VTE, the majority of patients were asymptomatic. However, in our study population symptoms caused by thoracotomy complicate the diagnosis of PE, therefore contributing to the underestimation of its true incidence. The clinical relevance of PE in patients undergoing thoracic surgery for cancer remains undefined.

Early diagnosis of PE is essential to establish adequate treatment to prevent recurrence.

$\mathrm{PE}$ in patients undergoing thoracic surgery for cancer has a multifactorial origin. Injury at the operative site might play a specific role. A long vascular stump might increase the risk of in situ thrombosis.

Other potentially harmful technical aspects are as follows: intimal dissection at the ligature sites, stenosis, and/or flow turbulence induced by excessive angulations of the vascular axis.

In conclusion, we observed a high incidence of $\mathrm{PE}$ in patients undergoing thoracic surgery for cancer despite prophylaxis. The intrinsic mechanisms of these findings should be further explored. Studies are required to identify the best prophylactic regimen in such a clinical setting.

\section{References}

1. Ziomek S, Read R, Tobler G. Thromboembolism in patients undergoing thoracotomy. Ann Thorac Surg. 1993;56:223-7.

2. Deslauriers J, Ginsberg RJ, Dubois P, et al. Current operative mortality associated with elective resection for lung cancer. Can J Surg. 1989; 32:335-9.

3. Kalweit G, Huwer H, Volkmer I, et al. Pulmonary embolism: a frequent cause of acute fatality after lung resection. Eur J Cardiothorac Surg. 1996; 10:242-7.

4. Chuang TH, Dooling JA, Connolly JM, et al. Pulmonary embolization from vascular stump thrombosis following pneumonectomy. Ann Thorac Surg. 1966;2:290-8. 